\title{
A ENFERMAGEM E O CUSTO COM OS MATERIAIS HOSPITALARES: UMA REVISÃO BIBLIOGRÁFICA
}

\author{
Vivian Schutz ${ }^{1}$, Bruno Teixeira de Siqueira²
}

RESUMO: Este estudo teve por objetivo descrever a produção científica acerca dos custos hospitalares a partir do enfoque dos materiais utilizados pela Enfermagem no ambiente hospitalar. A metodologia utilizada foi a revisão bibliográfica de publicações entre 2000 e 2009, em bases eletrônicas; foram encontrados 42 trabalhos, sendo que apenas três preencheram os critérios de seleção da pesquisa. A revisão apontou pouca produção bibliográfica referente a custos na área da Enfermagem. Como conclusão, pôde-se observar que, na prática capitalista, profissionais que geram lucros maiores são mais visados pelo mercado; ainda, o enfermeiro, ao apropriar-se de conhecimentos gerencias que possibilitem o uso consciente dos materiais e minimizem perdas, torna-se membro indispensável no contexto hospitalar.

PALAVRAS-CHAVE: Enfermagem; Administração de materiais no hospital; Custos hospitalares.

\section{NURSING AND THE COST OF HOSPITAL MATERIALS: A BIBLIOGRAPHICAL REVIEW}

\begin{abstract}
This study aimed to describe scientific literature production on the subject of hospital costs, focussing on the materials used in nursing in the hospital environment. The method used was a bibliographic review of publications from 2000 to 2009 in electronic databases; 42 works were found, of which only three fulfilled the research selection criteria. The review highlighted the low bibliographic production referring to costs in the area of Nursing. As a conclusion, one may observe that in capitalist practice, professionals who generate greater profits are sought after by the market; still, the nurse, by accepting managerial knowledge which permits the conscious use of materials and minimizes waste, can become an indispensable member in the hospital context.
\end{abstract}

KEYWORDS: Nursing; Hospital administration of materials; Hospital costs.

\section{LA ENFERMERÍA Y EL COSTE CON MATERIALES HOSPITALARES: UNA REVISIÓN BIBLIOGRÁFICA}

RESUMEN: Este estudio tuvo por objetivo describir la producción científica acerca de los costes hospitalares considerando el enfoque de los materiales utilizados por la enfermería en el ambiente hospitalar. La metodología utilizada fue la revisión bibliográfica de publicaciones entre 2000 y 2009, en bases electrónicas; fueron encontrados 42 trabajos, siendo que solamente tres obedecían a los criterios de selección de la investigación. La revisión apuntó poca producción bibliográfica sobre costes en el área de la Enfermería. Como conclusión, se puede observar que, en la práctica capitalista, profesionales que generan más lucros son más buscados por el mercado; así como el enfermero, al apropiarse de conocimientos gerenciales que posibiliten el uso consciente de los materiales y minimizen pérdidas, se vuelve miembro indispensable en el contexto hospitalar.

PALABRAS CLAVE: Enfermería; Administración de materiales en hospital; Costes hospitalares.

${ }^{1}$ Enfermeira. Doutora em Enfermagem. Professora do Departamento de Enfermagem Fundamental e do Programa de Pós-Graduação da Escola de Enfermagem Alfredo Pinto, da Universidade Federal do Estado do Rio de Janeiro-EEAP UNIRIO.

${ }^{2}$ Acadêmico de Enfermagem da EEAP UNIRIO.

\author{
Autor correspondente: \\ Vivian Schutz \\ Universidade Federal do Estado do Rio de Janeiro \\ R. Xavier Sigoud, 290 - 22290-240 - Rio de Janeiro-RJ-Brasil \\ E-mail:vschutz@gmail.com
}

Recebido: $19 / 04 / 10$

Aprovado: 02/02/11 


\section{INTRODUÇÃO}

O elevado custo com a saúde no Brasil e a dificuldade em financiá-la levam os prestadores, financiadores, autoridades e usuários dos serviços de saúde a se preocupar cada vez mais com estes aspectos, bem com a repercussão na qualidade desses serviços.

Vivemos em uma sociedade capitalista, na qual existe um valor agregado a todas as coisas. Seja em um objeto ou na realização de produção intelectual, estamos imersos em um mundo que se comunica por meio de trocas que têm um valor expresso monetariamente. As empresas geram seus lucros através da manipulação bem sucedida desses valores, que podem ser considerados como a soma parcial ou total de qualidade e produtividade, de emoção e de razão, de intuição e lógica, de capacidade empresarial e trabalho, de fé e persistência, de firmeza e equilíbrio, de vontade e $\operatorname{garra}^{(1)}$.

Nas organizações - e, neste contexto, se inserem as empresas de saúde - os empreendedores se colocam diante de um novo cenário de ordem econômica e social. Os negócios estão diferentes, mais competitivos, e os lucros estão mais difíceis de ser conquistados, exigindo mais dedicação, redução de desperdícios, diminuição de supérfluos e controle das atividades financeiras por todos os que prestam algum tipo de serviço para a empresa e que precisam dela para atender suas necessidades.

Atenta-se para a quantidade dos recursos necessários para que uma empresa de saúde funcione no mercado e, de um modo geral, devem ser utilizadas técnicas de planejamento, coordenação, supervisão e controle para que a gestão desses recursos seja realizada de forma a manter a sobrevivência da empresa.

$\mathrm{O}$ interesse em desenvolver esse estudo baseiase no fato de que a concorrência é intensa na área da saúde e que, para se obter uma posição vantajosa neste mercado, é necessário e importante que a empresa conheça a gestão de custos. Frente ao exposto, a Enfermagem, ao prestar a assistência direta e indireta à clientela, nas 24 horas em uma empresa hospitalar, trabalha amplamente com os recursos disponibilizados para o cuidado, bem como tem um forte elo na cadeia de comunicação com os demais membros da equipe prestadora de serviços. Ela se torna um importante agente na gestão destes custos na perspectiva de otimizar a saúde financeira da empresa, o que favorece condições positivas para subsidiar padrões satisfatórios de qualidade de assistência. Salienta-se que, no desenvolvimento do processo de trabalho em enfermagem, se trabalha com aspectos relacionados aos recursos físicos, humanos, financeiros e materiais.

Com o desenvolvimento das ações de cuidar em meio às condições socioeconômicas em que o país está inserido, resultante da política governamental vigente, saber gerenciar os recursos disponíveis tem sido a principal atividade das organizações, sejam familiares ou empresariais, como a instituição hospitalar. Portanto, para compreensão da gestão de custo, um dos pontos a serem considerados é o perfil do cuidado que é oferecido pela Enfermagem e o financiamento das ações de cada unidade dentro do Sistema de Saúde.

Gerenciar custos, dentro de orçamentos restritos, é manter equilíbrio entre despesas, custos e receitas, garantindo a sobrevivência da organização, podendo-se visualizar a unidade hospitalar como uma empresa ${ }^{(2)}$.

Buscando aproximação com a investigação acerca da temática de custos com materiais na área da Enfermagem, realizou-se uma revisão de literatura, com o objetivo de descrever a produção cientifica acerca dos custos, a partir dos enfoques em relação aos materiais que são utilizados pela Enfermagem no ambiente hospitalar. Para melhor entendimento dos conceitos que serão abordados, torna-se pertinente apresentá-los.

"Custo" representa os gastos relativos a bens ou serviços utilizados na produção de outros bens e serviços. Na cadeia produtiva em que se insere o enfermeiro, o serviço produzido é o cuidado realizado ao cliente e, dentro dessa cadeia, se observa que existem custos com recursos humanos, com todos os materiais (seringa, agulha, luvas e qualquer equipamento que venha a ser utilizado para produzir o cuidado), além daqueles com a estrutura hospitalar (espaço físico, luz e telefone), entre outros ${ }^{(3)}$.

Os custos podem ser classificados como diretos, indiretos ou de transformação. Custos diretos são aqueles diretamente incluídos no cálculo do produto, são os materiais e mão-de-obra diretos utilizados na prestação do cuidado, como os custos com salários dos enfermeiros e materiais como seringas, medicamentos e curativos. Custos indiretos necessitam de aproximação, algum critério de rateio para serem atribuídos ao serviço de cuidar, como aluguel do espaço, energia elétrica e água. Custo de transformação é o custo agregado pela instituição na obtenção do produto ${ }^{(3)}$.

Segundo a variabilidade, os custos podem ser classificados como fixos, variáveis, semifixos e semivariáveis. Custos fixos são custos que, em determinados 
períodos de tempo e em certa capacidade instalada, não variam, qualquer que seja o volume da atividade da empresa. Pode-se exemplificar com o aluguel do imóvel onde se instala o hospital: ele continuará existindo, não importando a quantidade de pacientes ali internados. Nos custos variáveis, seu valor se altera diretamente em função das atividades da empresa; são custos que irão variar de acordo com a produção, ao exemplo de medicamentos: se um hospital interna 50 clientes, terá que comprar medicamento para 50 pacientes; porém, se a internação for de 100 pacientes, terá que comprar medicação que supra as necessidades de 100 pacientes; com isso, o custo irá variar de acordo com as atividades da instituição. Os custos semifixos correspondem a custos que são fixos em determinado patamar, passando a ser variáveis quando esse patamar for excedido. Os custos semivariáveis correspondem a custos variáveis que não acompanham linearmente a variação da produção do volume de serviços; eles acompanham a variação aos saltos, mantendo-se fixos dentro de certos limites ${ }^{(3)}$.

"Despesas" correspondem a bens e serviços consumidos direta ou indiretamente para a obtenção de receitas. Não estão associadas à produção de um produto ou serviço; como exemplo de despesa, podem ser citados gastos com salários dos funcionários ${ }^{(3)}$.

"Gastos" consistem no sacrifício financeiro que a instituição arca para obtenção de um produto ou serviço qualquer. Eles poderão ser classificados como custos ou despesas, dependendo da sua importância na elaboração do produto ou serviço. Alguns gastos podem ser temporariamente classificados como investimentos e, à medida que forem consumidos, receberão a classificação de custos ou despesas ${ }^{(3)}$.

Para uma empresa, a obtenção do lucro é de grande importância. O lucro será o resultado das receitas subtraídas de custos e despesas ${ }^{(3)}$.

\section{METODOLOGIA}

Trata-se de revisão bibliográfica que empregou estudos primários da Literatura Latino-Americana e do Caribe em Ciências da Saúde (LILACS), Índice Bibliográfico Español en Ciencias de la Salud (IBECS), MEDLINE, Base de Dados de Enfermagem (BDENF) e bases bibliográficas brasileiras de teses e dissertações da Coordenação de Aperfeiçoamento de Pessoal de Nível Superior (CAPES). Foram utilizados os descritores: enfermagem, custos hospitalares, e administração de materiais no hospital. Os critérios de seleção foram: a) publicações entre os anos de 2000 e 2009; b) redigidos em português, inglês ou espanhol; c) tratar de custos hospitalares; d) dispor de texto completo.

O levantamento dos dados foi realizado no mês de março de 2010. Ao realizar a pesquisa cruzando os descritores enfermagem e custos hospitalares, foram encontrados 26 artigos, sendo 8 na base do LILACS, 16 na base do MEDLINE e 2 na base do BDENF.

Foi realizado o mesmo procedimento com os descritores enfermagem e administração de materiais no hospital. Foram encontrados 16 artigos, sendo 13 na base do LILACS, 1 na base do MEDLINE e 2 na base do BDENF. Ao total, foram encontrados 42 artigos.

Não foram encontradas teses ou dissertações com os descritores e critérios de inclusão propostos.

\section{RESULTADOS}

De posse dos 42 artigos coletados, procedeu-se à sua leitura, sendo que três atenderam aos critérios de seleção e constituíram a amostra para análise, a saber: "A enfermagem e o gerenciamento de custos"; "Classificação $\mathrm{ABC}$ dos materiais: uma ferramenta gerencial de custos" e "Glosas Hospitalares: importância das anotações de enfermagem".

Em um dos $\operatorname{artigos}^{(4)}$ foi abordada a questão dos custos hospitalares, contextualizando-os política e historicamente e mostrando os elementos que incentivam os profissionais a desenvolver estudos na área. O estudo mostra que o crescimento exponencial dos custos em saúde está diretamente relacionado a uma série de fatores, tais como: o emprego de novas tecnologias; o aumento da expectativa de vida da população; o crescimento da demanda, com a universalização do acesso à saúde; a escassez de mão-de-obra qualificada, acarretando baixa produtividade; a má gestão das organizações devido à incapacidade administrativa dos profissionais de saúde; a não-implantação de sistemas de controle de custos; e os desperdícios na cadeia produtiva, entre outros.

De acordo com outro artigo ${ }^{(5)}$, existe uma ineficiência do processo gerencial devido ao desconhecimento sobre custos de procedimento. $\mathrm{O}$ fato das organizações passarem a buscar ativamente o conhecimento de seus custos tem como fator, entre outros, a situação econômica brasileira. Na década de 1970, o país vivia um período de intenso crescimento econômico. Porém, na década 1980 houve o enfraquecimento marcado por uma crise mundial devido 
à elevação dos preços do petróleo, o que levou a uma instabilidade do sistema de preços, ocasionando a elevação da inflação. Já na década de 1990, houve a estabilização, porém muitas empresas fecharam e o índice de desemprego aumentou.

Em meio a este contexto, as empresas de saúde começam a se preocupar com o controle de custos. A atuação do enfermeiro é de gerir recursos humanos, materiais e financeiros, com vistas à eficiência na racionalização destes recursos de forma que sejam eficientes e financeiramente viáveis para a instituição, atentando para evitar desperdícios. Neste mesmo trabalho $^{(5)}$, utiliza-se a classificação $\mathrm{ABC}$ dos materiais, aplicando-a no contexto hospitalar para conhecer os gastos com os mesmos. Essa classificação pode ser definida como um instrumento a ser utilizado para o controle gerencial ${ }^{(3)}$; são destacados os materiais de elevado valor em relação aos outros: os itens A são aqueles cujos estoques apresentam elevado valor relativo e merecem um controle mais rigoroso que os demais; os itens B não são tão representativos como os estoques dos itens $\mathrm{A}$, mas representam elevada aplicação de recursos; e os do item $\mathrm{C}$ representam materiais que são expressivos em termos quantitativos, porém pouco representativos em termos de valor.

O texto enfatiza a importância do conhecimento, pela Enfermagem, sobre custos hospitalares. A classificação foi feita ordenando-se os itens de estoque segundo o seu Valor de Aquisição Ano (VAA) em ordem decrescente $(\mathrm{VAA}=$ quantidade adquirida $\mathrm{X}$ custo unitário); calculou-se o VAA acumulado item a item; calculou-se, para cada item, a percentagem do número de itens em relação ao valor total dos estoques; procedeu-se à divisão em classes.

Para classificar, utilizou-se o seguinte critério: itens de classe A representam 50\% dos custos, itens de classe B representam $30 \%$ e os de classe C $20 \%$, revelando que 67 itens representam os itens de classe A, 205 itens representam a classe B e 1666 representam a classe $\mathrm{C}$.

Um dos resultados obtidos pelo autor, e que é de grande importância para o enfermeiro, foi que os materiais assistenciais (como luvas, gazes, seringas, agulhas) representam $80 \%$ dos itens hospitalares e são os mais representativos na classe A. Esses materiais são utilizados, em sua maioria, pela equipe de enfermagem. Isto mostra a importância gerencial do enfermeiro na contenção dos custos hospitalares. Ao utilizar um método de classificação de materiais, o enfermeiro tem a capacidade de evidenciar quais são os materiais que necessitam de maior atenção e também lhe possibilita fazer um planejamento para aquisição desses materiais; a classificação possibilita um controle maior e melhor do estoque.

$\mathrm{O}$ outro artigo ${ }^{(6)}$ investiga os fatores que levam às glosas hospitalares*, observando que é de importância fundamental a anotação de enfermagem para evitá-las. Também aponta que os materiais utilizados no cuidado foram os que obtiveram o maior número de glosas. O trabalho foi realizado por meio de análise nas glosas ocorridas em uma determinada instituição e dos recursos elaborados pela mesma na tentativa de evitar que essas ocorressem. Ao todo, foram observados 82 tipos de glosas e um total de 17.324 itens glosados em um período de três meses. O componente material recebeu maior número de glosas $(86,1 \%)$, seguido do componente medicamento $(11,6 \%)$. Cabe ressaltar que ambos os componentes são constantemente utilizados pela equipe de enfermagem, que está em contato direto com os materiais e que é responsável pela administração dos medicamentos. $\mathrm{O}$ autor afirma que $\mathrm{o}$ volume de materiais não pagos pelos planos de saúde eleva de forma significativa os custos da instituição.

A pesquisa apontou que as anotações realizadas pela equipe de enfermagem têm fornecido informações suficientes e contribuído de forma substancial para um alto percentual de recuperação dos componentes glosados, o que evidencia a importância do profissional de enfermagem na questão financeira da instituição. No hospital em que ocorreu a pesquisa, os recursos de glosas totalizaram $\mathrm{R} \$ 31.856,52$, sendo $\mathrm{R} \$ 17.512,37$ em materiais e $\mathrm{R} \$ 10.099,42$ em medicamentos.

\section{DISCUSSÃO}

Apesar de ter atuação em todos os setores de uma empresa de saúde e lidar diretamente com os bens materiais desta, o enfermeiro ainda possui pouco entendimento relacionado à gestão financeira. Ao assumir a liderança ${ }^{(7)}$ de uma instituição de saúde, unidade de atendimento ou equipe de enfermagem, ele deve desenvolver novas habilidades, atitudes e conhecimentos para realizar algumas funções administrativas, entre as quais se ressalta a de coordenar o monitoramento de aspectos do controle orçamentário.

\footnotetext{
*Termo utilizado quando a seguradora se recusa a pagar a conta apresentada por entender que os procedimentos ou materiais utilizados não estão adequados ao tratamento
} 
Com isso, há a necessidade de uma transformação comportamental do enfermeiro, alicerçada na prática efetiva de uma nova cultura baseada na administração, tanto de recursos humanos quanto financeiros.

Os profissionais ${ }^{(5)}$ que gerenciam as unidades hospitalares devem desenvolver conhecimento que os auxilie nas tomadas de decisão e alocação de recursos, pois estes são geradores de custos dentro das organizações. Em qualquer circunstância dentro dos hospitais é sempre possível conter custos, seja na racionalização das atividades, no controle de materiais, redução de desperdícios, monitoramento e treinamento de suas equipes de trabalho. Logo, faz-se necessário que o enfermeiro desenvolva habilidades no manejo das variáveis econômicas, ambientais, tecnológicas e culturais de sua profissão.

Em decorrência da conjuntura econômica nacional, o setor privado de saúde enfrenta problemas financeiros e não consegue repassar seus aumentos de custos para o consumidor, devido à grande concorrência e à pressão da sociedade e dos planos de saúde. Já o setor público de saúde possui pouco investimento e recursos ${ }^{(4)}$. Por sua vez, os hospitais públicos ${ }^{(5)}$ de médio porte contam com aproximadamente 3.000 itens materiais; portanto, deve haver uma preocupação com o controle desse número de itens. Com esse cenário, tornou-se importante o conhecimento em gestão de custos.

Analisar os custos de uma empresa não significa que os valores dela devem estar baixos, mas sim que deve existir uma sincronia de valores e benefícios que esse custo irá gerar à empresa. É necessária uma análise do custo/benefício. Para manter-se no mercado competitivo ${ }^{(6)}$, as instituições têm que aprender a associar baixos custos com excelência de qualidade para seus clientes, e isso faz parte da gestão de custos, o que vai além das técnicas tradicionais de contabilidade, voltando-se para mudanças dos processos e para o atendimento das questões especiais relacionadas com a logística do atendimento correto aos clientes. Quanto maior a concorrência entre as instituições, mais importante se torna dispor de sistemas e trabalhadores que permitam a ela encontrar uma posição vantajosa diante de seus concorrentes, e o enfermeiro pode ser um profissional com grande diferencial para as empresas de saúde.

A eficiência de custos visa atender adequadamente a necessidade do serviço. Um material barato do qual não haja real necessidade da compra constitui um custo ineficiente; por outro lado, um material com custo elevado, mas que seja imprescindível, é um custo eficiente. A eficiência de custos pode ser definida como a obtenção do máximo com o dinheiro que se tem, ou a adequação do valor do produto ao seu preço ${ }^{(7)}$.

Evidenciou-se, nesta revisão, uma preocupação dos autores com o controle de custos. A auditoria é uma medida que visa o controle financeiro e a qualidade do atendimento. A auditoria é um exame sistemático e oficial do registro, do processo, da estrutura, do ambiente ou da contabilidade para avaliar o desempenho, o que pode utilizado como ferramenta importante para mensuração da qualidade (Auditoria de cuidados) e custos (Auditoria de custos) ${ }^{(7)}$.

A questão da auditoria dentro do estudo de custos tem a finalidade de controle tanto para o hospital quanto para as instituições que estarão custeando os serviços prestados ao cliente, como as seguradoras e planos de saúde. Um dos estudos ${ }^{(6)}$ evidenciou a ocorrência de glosa, em que o componente "material" recebeu o maior número delas, principalmente as compressas de gaze, as luvas cirúrgicas e o material para infusão (os mais evidenciados na pesquisa), seguido pelos medicamentos.

A questão do desperdício é outro fator que merece atenção por parte dos gestores da instituição, pois a falta de controle de material pode gerar prejuízos. Dessa forma, é necessário se valer de instrumentos gerenciais que possibilitem fazer controle de gastos sem que haja defasagem no processo produtivo, ou seja, na produção do cuidado. A implementação de sistemas gerenciais de custos é importante para a área da saúde, quando se visa a contenção de gastos sem a perda da qualidade do serviço ${ }^{(5)}$.

Como percebemos em um dos estudos realiza$\operatorname{dos}^{(5)}$, os materiais assistenciais utilizados pela Enfermagem representam sua maior parcela nos itens de classe A (em torno de $80 \%$ ), revelando que a luva de procedimento classificou-se como sétimo item de maior consumo na curva $\mathrm{ABC}$; assim, ressalta-se a importância do conhecimento gerencial de custos pela Enfermagem, tendo em vista que os materiais com maior consumo financeiro são justamente aqueles utilizados frequentemente pela equipe de enfermagem.

Diante do amplo número de agentes responsáveis pelo custo em saúde ${ }^{(4)}$, e entendendo o enfermeiro como um agente atuante nesse processo, há a necessidade de se estudar a inserção desses conteúdos nos cursos de formação de profissionais de nível médio profissionalizante de Enfermagem, de graduação e pós-graduação em Enfermagem. 


\section{CONSIDERAÇÕES FINAIS}

A revisão apontou um déficit na produção bibliográfica referente a custos dentro da área da Enfermagem. Essa defasagem contrapõe-se à importância do enfermeiro dentro da cadeia produtiva, tendo em vista que o enfermeiro é o profissional de maior contato com materiais da empresa. Por outro lado, e conforme apresentado nesta revisão, materiais de uso constante pela Enfermagem apresentam maior porcentagem no cálculo de custos hospitalares.

Cabe ressaltar que uma das atribuições do enfermeiro é o gerenciamento do ambiente físico e do material da unidade. Ele é o responsável pela manutenção do espaço e por controle de materiais; é de sua responsabilidade a fiscalização do uso desnecessário de materiais.

Na prática capitalista, profissionais que geram maiores lucros são mais visados pelo mercado. Ao se apropriar de conhecimentos gerencias que possibilitem o uso consciente dos materiais e minimizam desperdícios, o enfermeiro se torna mais valorizado para o mercado. Na ótica das instituições públicas de saúde, o recurso financeiro que se demanda para uma atividade dispendiosa e desnecessária irá fazer falta para gastos realmente essenciais.

\section{REFERÊNCIAS}

1. Pompermayer CB, Lima JEP. Gestão de custos. In: Mendes JTG, organizador. Finanças empresariais. Curitiba: Associação Franciscana de Ensino Senhor Bom Jesus/ FAE Bussines School/Gazeta do Povo; 2002. p.49-58.

2. Kurcgant P, coordenadora. Gerenciamento em enfermagem. Rio de Janeiro: Guanabara Koogan;2005.

3. Bruni AL, Fama R. Gestão de custos e formação de preços. $3^{a}$ ed. São Paulo: Editora Atlas; 2004.

4. Francisco IMF, Castilho V. A enfermagem e o gerenciamento de custos. Rev Esc Enferm USP. 2002;36(3):240-4.

5. Lourenço KG, Castilho V. Classificação $\mathrm{ABC}$ dos materiais: uma ferramenta gerencial de custos em enfermagem. Rev Bras Enferm. 2006;59(1):52-5.

6. Rodrigues VA, Perroca MG, Jericó MC. Glosas hospitalares: importância das anotações de enfermagem Arq Ciênc Saúde. 2004;11(4):210-4.

7. Marquis BL, Huston CJ. Administração e liderança em enfermagem: teoria e prática. $4^{\mathrm{a}}$ ed. Porto Alegre: Artmed; 2005. 